\title{
Exploring 3D User Interface Technologies For Improving The Gaming Experience
}

\author{
Arun Kulshreshth \\ Department of EECS \\ University of Central Florida \\ 4000 Central Florida Blvd \\ Orlando, FL 32816, USA \\ arunkul@knights.ucf.edu
}

\author{
Joseph J. LaViola Jr. \\ Department of EECS \\ University of Central Florida \\ 4000 Central Florida Blvd \\ Orlando FL 32816, USA \\ jj1@eecs.ucf.edu
}

\begin{abstract}
We present the results of a comprehensive video game study which explores how the gaming experience is effected when several 3D user interface technologies are used simultaneously. We custom designed an air-combat game integrating several 3DUI technologies (stereoscopic 3D, head tracking, and finger-count gestures) and studied the combined effect of these technologies on the gaming experience. Our game design was based on existing design principles for optimizing the usage of these technologies in isolation. Additionally, to enhance depth perception and minimize visual discomfort, the game dynamically optimizes stereoscopic 3D parameters (convergence and separation) based on the user's look direction. We conducted a within subjects experiment where we examined performance data and self-reported data on users perception of the game. Our results indicate that participants performed significantly better when all the 3DUI technologies (stereoscopic 3D, head-tracking and finger-count gestures) were available simultaneously with head tracking as a dominant factor. We explore the individual contribution of each of these technologies to the overall gaming experience and discuss the reasons behind our findings.
\end{abstract}

\section{Author Keywords}

Air-combat game; Finger-Count; Stereoscopic 3D; Head tracking; User Study; Video Games; Game Design; Game-play metrics; Player Behavior; User Experience.

\section{ACM Classification Keywords}

H.5.m Information Interfaces and Presentation (e.g. HCI): Miscellaneous.; K.8.0 Personal Computing: Games

\section{General Terms \\ Design, Experimentation, Measurement, Performance, Human Factors.}

\section{INTRODUCTION}

3D user interface technologies [4] (e.g., stereoscopic 3D, head tracking, gesture based control, etc.) have the poten-

Permission to make digital or hard copies of all or part of this work for personal or classroom use is granted without fee provided that copies are not made or distributed for profit or commercial advantage and that copies bear this notice and the full citation on the first page. Copyrights for components of this work owned by others than ACM must be honored. Abstracting with credit is permitted. To copy otherwise, or republish, to post on servers or to redistribute to lists, requires prior specific permission and/or a fee. Request permissions from permissions@acm.org.

CHI 2015, April 18 - 23, 2015, Seoul, Republic of Korea

Copyright (c) 2015 ACM 978-1-4503-3145-6/15/04...\$15.00

http://dx.doi.org/10.1145/2702123.2702138

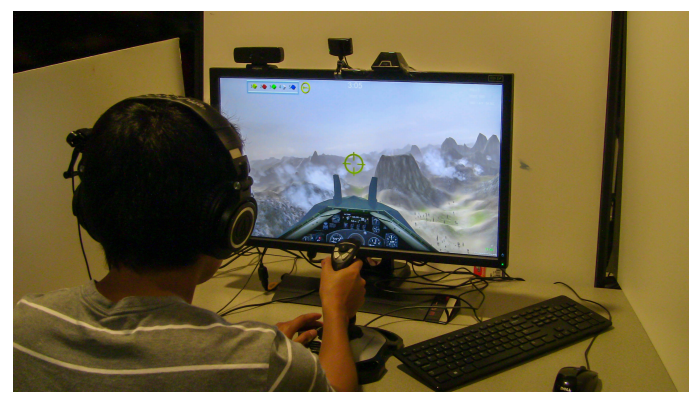

Figure 1. A user playing the air-combat game we designed. The game effectively uses stereoscopic 3D, head tracking and finger-count gestures.

tial to improve game performance and the gaming experience. Such interfaces allow users to use natural motion and gestures to control the game thereby making the whole gaming experience more immersive and engaging. In the past, researchers $[9,10,11,18,19]$ have studied the benefits of these technologies (e.g. stereoscopic 3D, head tracking, gesture based control, etc.) for video games. But, most of the past work have been focused on these technologies in isolation and it is still unknown how the gaming experience will be affected if several 3DUI technologies are used simultaneously. By designing a game which integrates several 3DUI technologies, we hope to understand the interplay between the technologies and its effect on the gaming experience.

Stereoscopic 3D and head tracking are two core technologies for 3DUI applications. Stereoscopic 3D [11, 18, 19] and head tracking $[10,21,22]$, in isolation, have been shown to provide a better gaming experience along with performance benefits for some games. Based on these findings, we chose to use stereoscopic 3D and head tracking for our game design. We chose to design an air-combat game since it had tasks (e.g. judge the distance of an enemy, find enemy, etc.) which could benefit from the availability of stereoscopic 3D and head tracking usage. Furthermore, to enhance depth perception and minimize visual discomfort, our game dynamically adjusts the stereoscopic 3D parameters (convergence and separation), based on the user's look direction.

Additionally, we wanted to include a 3DUI input mechanism in our game to create a more inclusive 3D user interface experience and chose a gesture-based interface. Initially, we experimented with several motion sensing devices (e.g. Leap Motion, Microsoft Kinect, etc.) but these devices failed for our purposes for two reasons. First, the gestures recognition 
accuracy of these devices was not good enough for precisely controlling the aircraft in our game. Second, users needed to continuously control the airplane causing fatigue during our pilot testing sessions (lasting for about 100 minutes). These factors hindered the overall gameplay experience. However, finger-count gestures [9] are well studied in the past and have higher recognition accuracy as well as being easy to use and fast to perform. These gestures could potentially be used as shortcuts in video games. The finger count gestures were well suited for longer use since the user is not using them continuously while playing the game. Therefore, we used these gestures as an alternate to using buttons for switching weapons. We refer to them as finger-count shortcuts.

In this paper, we designed an air combat game (see Figure 1) and conducted a within subjects experiment to evaluate the effectiveness of simultaneous use of these technologies (stereoscopic 3D, head tracking and finger-count shortcuts). We examined the performance data (enemies killed \& survival time), head tracking usage data, and self-reported data on user's perception of the game.

\section{RELATED WORK}

Stereoscopic 3D has been found useful for games depending upon the game task involved. Stereoscopic 3D has also been found to be helpful in playing simple games where a user is manipulating a single object at a time [6]. Rajae et al. [16] showed that presence of stereoscopic 3D did not help people perform better in a shooter game but people experienced a stronger feeling of presence in stereoscopic 3D mode. Other researchers $[13,18,19]$ have also confirmed increased engagement and preference for stereoscopic 3D games.

Creating graphical user interfaces (GUI) for stereoscopic 3D games is a difficult choice between visual comfort and effect. Schild et al. [17] explored GUI design space for a stereoscopic 3D game in order to design comfortable game GUIs (e.g., menus and icons). Their results showed that ingame menus look best when displayed at the bottom of screen with a semi-transparent background. For referencing objects, they found that it is best to show the referencing information at the same depth as the object itself. Deepress3D is a flight game [18] which was custom designed keeping stereoscopic 3D viewing in mind. Their game design featured a stereoscopic specific GUI based on [17] , no real depth illusions in graphics, and optimal parallax budget for stereoscopic viewing. Their results show that the users experienced an enhanced sense of presence in the stereoscopic 3D viewing environment.

Stereoscopic 3D benefits can only be expected if the stereoscopic vision is not accompanied by distortions (e.g., contradicting depth cues, ghosting/cross-talk, exaggerated disparity) [27]. While stereoscopic 3D has shown some positive benefits depending on the task, it also has shown to cause negative symptoms as well, such as eyestrain, headache, dizziness, and nausea [8]. Ware [25] proposed dynamic adjustment of stereoscopic parameters to minimize visual discomfort and optimize stereo depth. Our game design also dynamically adjusts stereoscopic 3D parameters (convergence and separation), based on user's look direction, for a comfortable viewing experience and still enhance stereo depth perception whenever possible.

Wang et al. [24] used face tracking for head gesture recognition. Their evaluation, based on simple game prototypes they developed, showed that the test participants experienced a greater sense of presence and satisfaction with their head tracking technique. However, no performance benefits were found compared to a button based head control. Yim et al. [26] developed a head tracking solution using Wiimotes and their preliminary results show that users perceived head tracking as a more enjoyable and intuitive gaming experience. Sko et al. [21] explored head tracking for first person shooter games. Their study showed that head tracking could be useful for games which are designed with head tracking usage in mind. Kulshreshth et al. [10] explored head tracking for a variety of games and found it to be useful for a few games depending upon the game type and the gaming experience of the participants. However, they used commercial games for their experiment and some of their games may not have been designed with head tracking usage in mind, possibly explaining why they did not find performance benefits in all the games they tested.

For optimal head tracking usage in a game some design guidelines have been proposed [10,22] which includes proper training for head tracking usage, avoiding awkward head movements, non-isomorphic head rotations with different scaling along different directions, natural movements for tasks and proper calibration of head tracking device. For our air-combat game, we made use of all these design principles to optimize head tracking usage.

Barfield et al. [3] studied the effects of stereoscopic 3D and head tracking on a wire-tracing task. Their results indicated that the task time was the same irrespective of display conditions (monoscopic vs stereoscopic 3D) when head tracking was present. People performed best with stereoscopic 3D when head tracking was absent. McMahan et al. [14] explored the interplay between display fidelity and interaction fidelity. Their results showed that the performance was best with low-display low-interaction fidelity and high-display and high-interaction fidelity. Another experiment involving a spatial judgment task [15] showed that the participants performed better with head-tracking and best performance was achieved when both stereoscopic 3D and head tracking was present. The worst score was achieved with a combination of monoscopic display and no head tracking. However, none of these researchers used complex video games for their experiments.

Finger-Count menus were first proposed for multi-touch surfaces [1] and were later adapted for distant displays [2], using the Kinect as the gestural input device. But, the Kinect based implementation was slow for practical use. Another implementation [9] improved the selection time of this technique by using a faster finger recognition algorithm resulting in an average selection time of under a second. Their study showed that Finger-Count shortcuts have a high accuracy and are layout independent. Finger-Count gestures could also be used as shortcuts in video games. Our game these finger-count shortcuts for switching between weapons. 


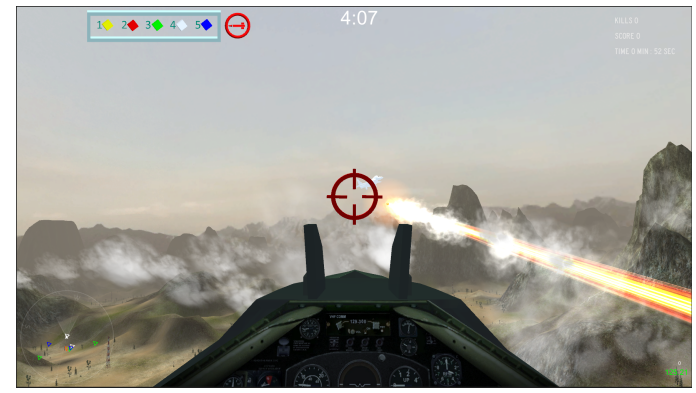

Figure 2. Air-combat game screenshot

None of the work mentioned above evaluated the affects of using several 3DUI technologies together in complex gaming environments like in modern video games. To the best of our knowledge, our work is the first to systematically explore the combined affect of several 3DUI technologies in a custom designed game with several design optimizations specific to each technique.

\section{DESIGN FACTORS}

As mentioned in the introduction, we chose to design an aircombat game since it had tasks which could benefit from availability of stereoscopic 3D, head tracking usage, and finger-count gestures. Furthermore, an air-combat game scene has a lot of depth and using stereoscopic 3D would make the game more immersive. The design of this game includes several optimizations, based on past work, specific to stereoscopic 3D $[11,17,18,20]$ and head tracking $[10,21$, 22].

\section{Air-Combat Game}

The player has to control an aircraft, using the Logitech extreme 3D Pro joystick, in first person view and shoot enemies (see Figure 2 for a screenshot of the game). The game has five different kind of enemies, each marked with a different color, and five different kind of weapons. The color of the crosshair indicates the color of the currently selected weapon. Each enemy can be killed only with a weapon of the same color and thus requires a user to frequently switch weapons while playing the game. A radar is also available which shows $2 \mathrm{D}$ positions of the enemies around the aircraft. To be consistent with the color scheme, the radar uses the same color as the enemy to display its position. The game also featured 3D sound effects for aircraft, weapons and explosions (when enemies are shot dead). An enemy could also be locked (except for yellow and green enemies) by holding the crosshair over it for a short period of time (about two seconds).

The head of the player can be controlled either by using head tracking (a TrackIR 5 device was used) or a combination of the hat switch and buttons on the joystick (see Figure 3). To switch weapons one can use finger-count shortcuts or buttons on the joystick (one button is assigned for each weapon). To avoid any confusion each button is clearly marked with a color on the joystick. In case of finger-count shortcuts, a chart was displayed at the top of the screen indicating the correspondence between finger-count gestures and weapon colors. The game was implemented using the Unity3D game engine and the Air Strike Starter Kit from the Unity Asset Store. For

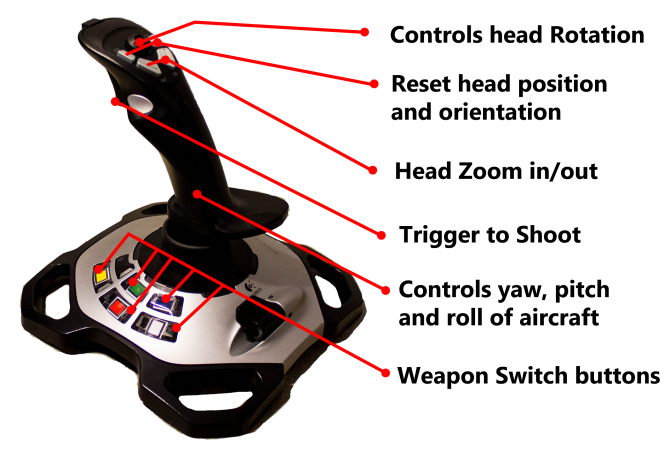

Figure 3. Joystick Controls for the air-combat game

implementing finger-count shortcuts, we used the Intel's perceptual computing SDK.

\section{Stereoscopic 3D features}

Dynamic Stereo. Currently, most stereoscopic 3D games fix convergence and separation values for optimal depth budget throughout the game. But, this approach reduces stereo depth when a large object (e.g. gun in FPS games, cockpit in aircombat, etc.) is present in front of the game camera. The reason being the fact that stereo parameters have to be optimized to keep that large object always in focus. However, when the player's head is rotated, that nearby object is not in the players view and stereo depth could be increased. In case of our aircombat game, we optimized stereo parameters under two conditions. First, when the user is looking sideways (left/right) and second, when the user is zoomed into the scene. In both these cases, the user is not looking at the cockpit in front. When the player's head is rotated sideways (left/right), the separation is increased with linear scaling proportional to the heads rotation and the convergence is not changed. When a user zooms in the scene the field of view (FOV) of the camera is reduced proportional to the head's displacement. Thus, in case of zooming, the separation is increased with linear scaling proportional to the camera's FOV. At the same time, the convergence is linearly decreased with the camera's FOV to keep both the crosshair and background in focus. These dynamic parameters ensured a comfortable stereoscopic 3D experience and provided better depth perception for this aircombat game.

Stereoscopic 3D Specific GUI Elements. Based on [17], we optimized our game GUI for stereoscopic 3D usage. All the 2D GUI elements (timer, game stats, etc.) were rendered at screen depth to allow them to be in focus throughout the game. The radar was displayed at the bottom of the screen and was also rendered at screen depth. The chart displaying the correspondence between finger-count gestures and weapon colors was a 3D object rendered at the same depth as the aircraft to be visible all the time without being occluded by other 3D objects in the scene.

Optimal Depth Cues. The game minimized the impact of monocular depth cues. All the enemy ships were colored instead of textured. No dynamic light sources were used and shadows (a known depth cue) were disabled. 
Disable Post-processing Image Effects. Some postprocessing image effects (e.g. halo effect for lights) do not work well with stereoscopic $3 \mathrm{D}$ rendering since these effects are rendered only for one eye making it uncomfortable to look at. Hence, we did not use any post-processing image effects for our game.

Minimized 3D Glasses Flicker. Excessive motion in front of the display may sometime cause the 3D glasses to flicker due to loss of sync signal [11]. In our case head tracking was used only for head rotations \& zooming and all other motions were restricted. In case of head rotation, the head position does not change and the head rotation is also limited (about 40 degrees each side). When a user zooms in, the head moves towards the Nvidia IR emitter. Thus, in both these cases the head motion is minimal and does not interfere with $3 \mathrm{D}$ sync signal loss. Furthermore, we noticed that Nvidia 3D vision 2 glasses were flickering when used together with the Creative Senzeye3D depth camera (used for detecting fingercount gestures). We suspect that there was some interference between IR blaster inside the camera and the 3D sync signal from Nvidia IR emitter causing the glasses to loose sync signal. However, older Nvidia 3D vision glasses worked fine without any flickering issues. Hence, we used older Nvidia 3D vision glasses instead of newer 3D vision 2 glasses for our experiments.

\section{Head Tracking Features}

Natural Head Movements. People are used to rotating their head for looking around. We mapped head tracking to use these natural movements for looking through the sides of the plane and zooming in. Thus, it is very easy to understand the head tracking usage for our air-combat game.

Adaptable Setup. Since every user is different (in terms of height/size and comfortable sitting position), the starting head position in the game was customized for each user. We asked users to sit in their relaxed pose and that was chosen as the starting head position/orientation. The user's motion is then detected relative to that starting pose. Thus, we ensured that each user is comfortable while playing the game.

Training for Head Usage. A prior experiment on head tracking usage in video games [10] found that experienced gamers make better use of head tracking than casual gamers. Casual gamers pay more attention to learning how to play the game and do not use these extra features to their advantage. To avoid this problem, we trained all our participants, irrespective of their gaming experience, to be able to play the game and use head tracking at the same time.

Avoid Awkward Head Movements. We restricted the player's head position/orientation to avoid most awkward head poses. The player's head could only be rotated sideways (left/right) and up/down. The head position was fixed along axes parallel to the display to allow only one directional movement toward display while zooming. These restrictions ensure that the users don't get disoriented while playing thereby reducing head-tracking based motion sickness (nausea).

Non-Isomorphic Head Rotations. When users are looking at the display, they can not rotate their head beyond a certain range depending upon the display size and the player's distance from display. In the past, non-isomorphic rotations seem to have helped in rotation tasks [12] when head tracking is present. We used non-isomorphic rotation scaling for left and right rotations to allow users to see more area on both sides of the plane without rotating his head too much. We thought this would help them quickly scan a large area of the game environment for finding potential enemies.

\section{Why Five Enemies and Five Weapons?}

As part of our experiment, we wanted to evaluate the performance of finger-count shortcuts, as a fast way to switch weapons, compared to buttons. Since the user were using one hand to control the plane, only one hand was available for finger-count gestures. This limits the number of finger-count gestures to five. This motivated us to keep five different kind of enemies. Moreover, we wanted people to use these gestures frequently throughout the game play session. Thus, we designed five different kind of weapons and added a restriction that each enemy can be killed only by a specific weapon.

\section{USER EVALUATIONS}

We conducted an experiment with our air-combat game to evaluate the combined effect of stereoscopic 3D, head tracking, and finger-count shortcuts on the gaming experience. Additionally, we also looked at the effects of individual technologies to be able to understand their contribution to the overall gaming experience. Based on previous findings in related work and our analysis of the game, we have the following hypotheses:

Hypothesis 1 (H1) : The combined usage of stereoscopic 3D, head tracking and finger-count shortcuts improves user's gaming performance compared to the control condition with monoscopic display, no head tracking and buttons for weapon switching.

Hypothesis 2 (H2) : Stereoscopic 3D improves user's gaming performance compared to the monoscopic display condition.

Hypothesis 3 (H3) : Head-tracking improves user's gaming performance compared to button based head control.

Hypothesis 4 (H4) : User's performance with finger-count shortcuts will be same as with buttons.

Hypothesis 5 (H5) : Participants prefer to use Finger-count shortcuts compared to buttons.

\section{Subjects and Apparatus}

We recruited 32 participants (29 males and 3 females ranging in age from 18 to 30 with a mean age 20.84) from the university population, of which four were left handed. Out of all participants, only 4 had prior experience with head tracked games, 8 had played stereoscopic 3D games, and 30 people had played motion controlled games. The experiment duration ranged from 100 to 120 minutes and all participants were paid $\$ 10$ for their time.

The experiment setup, shown in Figure 4, consisted of a 27" BenQ XL2720Z 3D monitor, Nvidia 3D Vision kit, a TrackIR 5 with Pro Clip (mounted on a headphone), a Creative Senz3D depth camera, a Logitech Extreme 3D Pro joystick, and a PC (Core i7 4770K CPU, GTX 780 graphics card, 


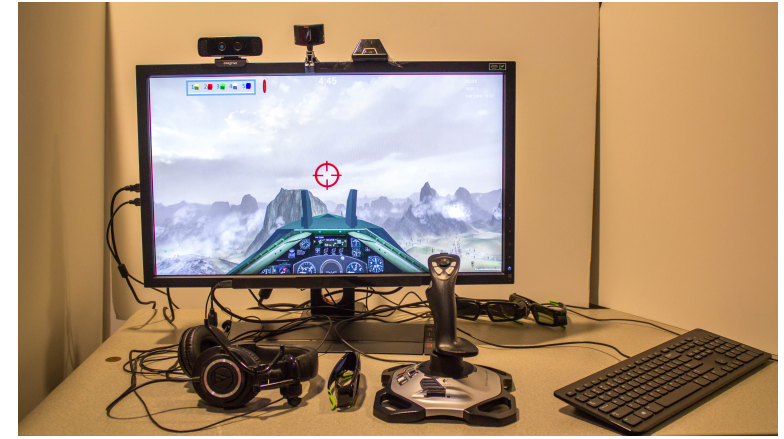

Figure 4. The Experimental Setup.

8 GB RAM). We used the Unity3D game engine and Intel Perceptual Computing Software Development Kit (PCSDK) for implementing the game. The TrackIR 5 camera, the creative camera, and the Nvidia IR emitter were mounted on the top of monitor. Participants were seated about 2 feet away from the display. Since the 3D Vision glasses could impact the subjective feel and comfort level of the participants under different condition, participants were asked to wear them throughout the experiment. In non-stereoscopic condition, the open shutters of the glasses provide an image slightly darker than without glasses but minimally brighter than the stereoscopic 3D version. To make sure that all our participants are able to see stereoscopic 3D, we used Nvidia medical test image to test stereo abilities of participants. All our participants passed the test. All participants preferred using their right hand (despite some of them being left handed people) for joystick control and left hand for weapon switching (buttons or finger-count gestures).

\section{Experiment Design and Procedure}

We chose a within-subjects design for our experiments in order to be able to measure and compare user perceptions of the game on a variety of quantitative and qualitative metrics. This within-subjects experiment had 3 independent variables: display mode (Stereoscopic 3D and monoscopic 2D), head control mode (head-tracked and button based head control) and weapon switch mode (finger-count shortcuts and buttons). In total we had $2 \times 2 \times 2=8$ conditions and for each condition the user conducted two trials which makes a total of 16 game plays per participant as part of this experiment. Each game trial ends if the player dies (if hit with another plane or ground, shot by another plane) or if the time limit of $5 \mathrm{~min}$ utes is reached. Our dependent variables were mean survival time and mean number of enemies killed, where the mean is taken over the two trials for that condition.

The experiment began with the participant seated in front of the monitor and the moderator seated to the side. Participants were given a consent form that explained the experiment procedure. They were then given a modified version of Terlecki and Newcombe's video game experience survey [23] as a prequestionnaire which collected general information about the participant (age, sex, dexterity) and their prior gaming experience. At the beginning, each participant was trained for about 20-25 minutes on how to play the game under different experimental conditions. Participants then played the game under

\begin{tabular}{|l|l|}
\hline \multicolumn{2}{|c|}{ Game Questions } \\
\hline Q1 & To what extent did the game hold your attention? \\
\hline Q2 & How much effort did you put into playing the game? \\
\hline Q3 & How mentally demanding was this game? \\
\hline Q4 & Did you feel hurried or rushed when playing this game? \\
\hline Q5 & To what extent you felt frustrated while playing? \\
\hline Q6 & To what extent did you find the game challenging? \\
\hline Q7 & To what extent did you enjoy the graphics and the imagery? \\
\hline Q8 & $\begin{array}{l}\text { To what extent you felt that you were part of the game rather } \\
\text { than just observing? }\end{array}$ \\
\hline Q9 & $\begin{array}{l}\text { To what extent you felt that you were physically present in } \\
\text { the game environment presented? }\end{array}$ \\
\hline Q10 & How much would you say you enjoyed playing the game? \\
\hline Q11 & Would you like to play the game again? \\
\hline Q12 & $\begin{array}{l}\text { Which aspects of the game made your overall game experience } \\
\text { better? Stereoscopic 3D, Head-tracking, Finger-count shortcuts? }\end{array}$ \\
\hline
\end{tabular}

Table 1. Post-Questionnaire. Participants responded to question 1-11 on a 7 point Likert scale. Question 12 was a multiple choice question.

each condition. Each condition presented to the user in random order based on a Latin square design [5]. We recorded survival time, number of enemies killed and head tracking usage data for each gaming condition presented during experiment. After the experiment, the participant filled out a postquestionnaire with questions about their experiences with the game (see Table 1) including questions about stereoscopic 3D (see Table 2), head tracking (see Table 2), and finger-count shortcuts (see Table 3).

\section{RESULTS}

To analyze the performance data, we used repeated-measures 3-factor ANOVA per dependent variable. We did a post-hoc analysis using pairwise sample t-tests. We used Holm's sequential Bonferroni adjustment to correct for type I errors [7] and the Shapiro-Wilk test to make sure the data was parametric. To analyze the Likert scale data, we used Friedman's test and then a post-hoc analysis was done using Wilcoxon signed rank test. For all of our statistical measures, we used $\alpha=0.05$.

\section{Quantitative Results}

Repeated measures 3-factor ANOVA results are shown in Table 4. In terms of enemies killed, significant interactions were found based on the combined usage of all the three technologies $(\mathrm{DM} \times \mathrm{HCM} \times \mathrm{WSM})$. People killed significantly more $\left(t_{31}=-2.546, p<0.02\right)$ enemies when stereoscopic $3 \mathrm{D}$, head tracking and finger-count shortcuts $(\bar{x}=18.21, \sigma=$ $5.70)$ were present compared to a condition with monoscopic display, no head tracking and buttons for weapon switch $(\bar{x}=15.32, \sigma=4.88)$. There was no significant difference in survival time between the above two conditions.

We found significant differences in the number of enemies killed $\left(F_{1,31}=14.264, p<0.005\right)$ and the survival time $\left(F_{1,31}=14.215, p<0.005\right)$ based on the head control mode (NHT vs HT). Participants killed significantly more enemies $\left(t_{31}=-3.777, p<0.005\right)$ and survived significantly longer $\left(t_{35}=-3.770, p<0.005\right)$ when head tracking was present. 


\begin{tabular}{|c|c|c|}
\hline Source & Enemies Killed & Survival Time \\
\hline $\mathrm{DM}$ & $F_{1.31}=0.876, p=0.357$ & $F_{1.31}=0.021, p=0.886$ \\
\hline $\mathrm{HCM}$ & $F_{1.31}=14.264, p<0.005$ & $F_{1.31}=14.215, p<0.005$ \\
\hline $\mathrm{WSM}$ & $F_{1.31}=5.320, p<0.05$ & $F_{1.31}=3.255, p=0.081$ \\
\hline $\mathrm{DM} \times \mathrm{HCM}$ & $F_{1.31}=0.103, p=0.751$ & $F_{1.31}=0.932, p=0.342$ \\
\hline $\mathrm{DM} \times \mathrm{WSM}$ & $F_{1.31}=2.601, p=0.117$ & $F_{1.31}=1.791, p=0.191$ \\
\hline $\mathrm{HCM} \times \mathrm{WSM}$ & $F_{1.31}=3.705, p=0.063$ & $F_{1.31}=0.995, p=0.326$ \\
\hline $\mathrm{DM} \times \mathrm{HCM} \times \mathrm{WSM}$ & $F_{1.31}=6.221, p<0.05$ & $F_{1.31}=0.009, p=0.924$ \\
\hline
\end{tabular}

Table 4. Repeated measures 3-factor ANOVA results. DM: Display Mode, HCM: Head Control Mode, WSM: Weapon Switch Mode

\begin{tabular}{|c|l|}
\hline \multicolumn{2}{|c|}{ Stereoscopic 3D/Head Tracking Questions } \\
\hline Q1 & $\begin{array}{l}\text { To what extent did 3D/HT improved the overall experience of the } \\
\text { game? }\end{array}$ \\
\hline Q2 & To what extent 3D/HT was helpful in the game? \\
\hline Q3 & I would choose to play with 3D/HT over normal viewing. \\
\hline Q4 & I felt that 3D/HT enhanced the sense of engagement I felt. \\
\hline Q5 & 3D/HT is a necessity for my future game experiences. \\
\hline Q6 & $\begin{array}{l}\text { To what extent you felt that the head tracking helped you find } \\
\text { enemies in the environment faster? }\end{array}$ \\
\hline Q7 & Do you feel that 3D/HT helped you to perform better? \\
\hline Q8 & How did 3D/HT help you perform better? \\
\hline Q9 & Do you feel that 3D/HT hurt your performance? \\
\hline Q10 & How did 3D/HT hurt your performance? \\
\hline Q11 & $\begin{array}{l}\text { Did you feel any Symptoms from viewing the games in stereo- } \\
\text { scopic 3D (eye strain, headaches, dizziness, Nausea)? }\end{array}$ \\
\hline
\end{tabular}

Table 2. Stereoscopic 3D/Head Tracking Questionnaire. Participants responded to question 1-6 on a 7 point Likert scale. Questions 7-10 were multiple choice and open ended questions to gauge the users perception of the effects of stereoscopic 3D. In question 11, each symptom had a 7 point Likert scale to indicate the extent of each symptom ranging from not at all to very much so.

Mean number of enemies killed and mean survival time under different gaming conditions is shown in Figure 5 and Figure 6 respectively. There was no significant difference in the number of enemies killed or the survival time based on the display mode (2D vs 3D). Compared to monoscopic mode, people killed slightly more enemies when stereoscopic 3D was present. We found significant differences in terms of enemies killed based on the weapon switch mode (buttons vs finger-count shortcuts) but no significance was found in the post-hoc analysis.

Furthermore, the gaming experience of the participants did not play a significant role in the performance of the participants across different gaming conditions. The statistics were same even when we divided the participants in two groups, casual and experienced, and compared performance data for all gaming conditions separately for two groups.

To compare our results with prior research [3, 15], we also looked at the number of enemies killed and the survival times based on only the display mode and the head tracking mode (see Figure 7). We found that people killed most enemies when both head tracking and stereoscopic 3D was present but survival time was slightly more for head tracked (HT) and monoscopic condition. Overall, head tracking played a significant role in performance and stereoscopic 3D had only minor performance impact.

\begin{tabular}{|c|l|}
\hline \multicolumn{2}{|c|}{ Finger-Count Questions } \\
\hline Q1 & $\begin{array}{l}\text { To what extent did the finger-count gestures improved the overall } \\
\text { experience of the game? }\end{array}$ \\
\hline Q2 & $\begin{array}{l}\text { To what extent did you feel that the finger-count gestures were } \\
\text { helpful while game play? }\end{array}$ \\
\hline Q3 & $\begin{array}{l}\text { To what extent do you think that using a finger-count for weapon } \\
\text { switch was better than using buttons? }\end{array}$ \\
\hline Q4 & $\begin{array}{l}\text { The finger-count gestures hurt your performance in the tasks that } \\
\text { were presented? }\end{array}$ \\
\hline Q5 & $\begin{array}{l}\text { Do you feel that the finger-count gestures should be used for fu- } \\
\text { ture games? }\end{array}$ \\
\hline Q6 & $\begin{array}{l}\text { Are there any other game tasks (not specific to this game) where } \\
\text { finger-count shortcuts could be used? }\end{array}$ \\
\hline
\end{tabular}

Table 3. Finger-Count Questionnaire. Participants responded to question 1-3 on a 7 point Likert scale. Questions 4 and 5 were yes/no questions. Question 6 was an open ended question.

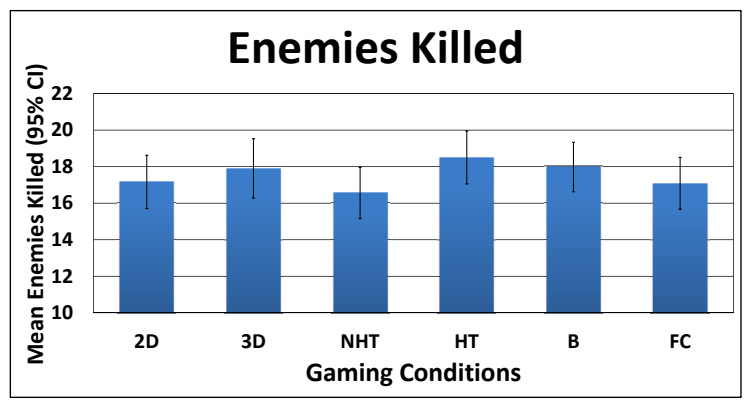

Figure 5. Mean number of enemies killed under different gaming conditions where 2D : Non-stereoscopic 3D, 3D: Stereoscopic 3D, NHT: Nonhead-tracked, HT: Head-tracked, B: Button based weapon switch, and FC: Finger-Count based weapon switch.

When head control data (see Figure 8) was analyzed, we found that significantly more people, compared to button based head control, used head controls when head tracking was present $\left(t_{31}=6.917, p<0.005\right)$. We also found that people use zoom (iron sight) more often compared to looking sideways (left/right).

Mean ratings for game post-questionnaire questions 1 to 11 (see Table 1) are summarized in Figure 9. We can see that:

- The game held attention of all the participants and everyone tried their best.

- The game had moderate mental demand and difficulty level.

- Participants did not feel frustrated while playing and indicated that they would like to play the same game again. 


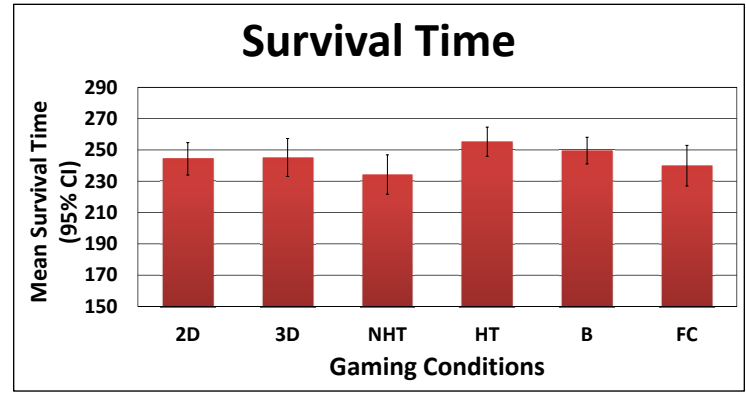

Figure 6. Mean survival time under different gaming conditions where 2D : Non-stereoscopic 3D, 3D: Stereoscopic 3D, NHT: Non-headtracked, HT: Head-tracked, B: Button based weapon switch, and FC: Finger-Count based weapon switch. Higher survival time is better.

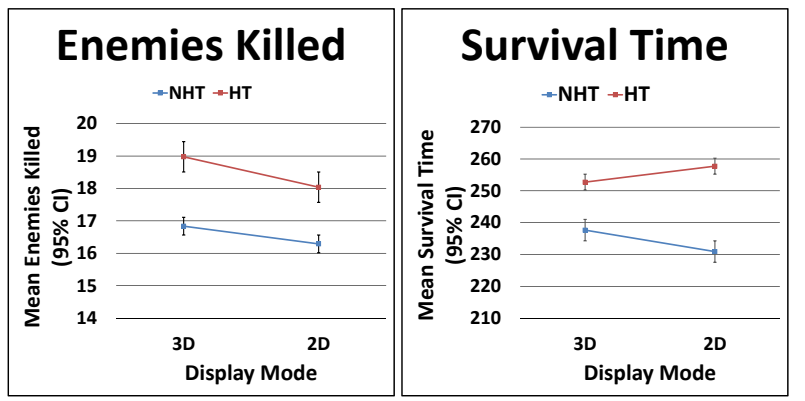

Figure 7. Mean enemies killed and mean survival time irrespective of weapon switch mode where 2D : Monoscopic, 3D: Stereoscopic 3D, NHT: Non-head-tracked mode, and HT: Head-tracked mode.

- Graphics quality of the game was rated as high and people enjoyed the game.

Out of 32 participants, 27 liked head tracking, 22 liked stereoscopic 3D and only 11 liked using finger-count shortcuts (see Figure 10). Majority of participants thought that stereoscopic $3 \mathrm{D}$ and head tracking was helpful in the game. Four people thought that stereoscopic 3D hurt their performance and five people thought that head tracking hurt their performance. People were divided about their views on finger-count shortcuts. Out of 32, 17 thought that finger-count shortcuts helped them perform better and 15 thought that it hurt their performance. Nineteen people thought that finger-count gestures should be used for future games.

Users perception of the three technologies revealed some interesting findings. Mean ratings for stereoscopic 3D, head tracking, and finger-count post-questionnaire questions (Q1Q5 for 3D \& HT, Q1-Q3 for FC) are summarized in Figure 11. Overall experience was significantly $\left(\chi^{2}=11.327, p<\right.$ $0.005)$ different across three technologies. Head tracking provided significantly better $(Z=-2.693, p<0.01)$ overall experience compared to finger-count shortcuts. Helpfulness of technologies was significantly different $\left(\chi^{2}=\right.$ $7.856, p<0.05)$ across technologies. Head tracking was significantly more $(Z=-2.339, p<0.02)$ helpful than finger-count shortcuts. Preference ratings of the technologies were also significantly different $\left(\chi^{2}=6.018, p<0.05\right)$. Head tracking $(Z=-2.249, p<0.03)$ and stereoscopic 3D $(Z=-2.125, p<0.04)$ had significantly higher preference rating than finger-count shortcuts. There was no signif-

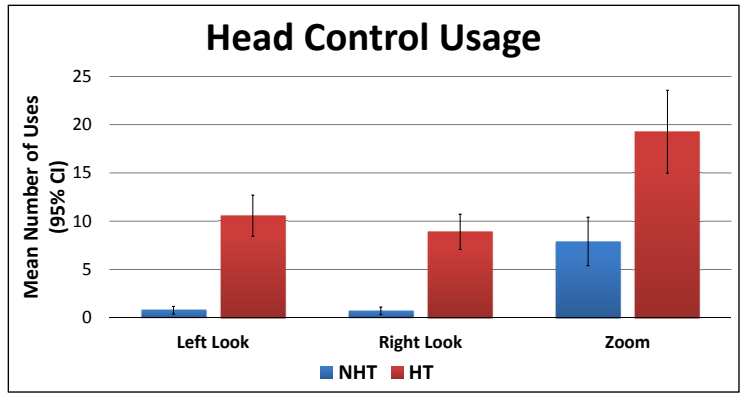

Figure 8. Players head control usage based on head control mode (buttons vs head tracking)

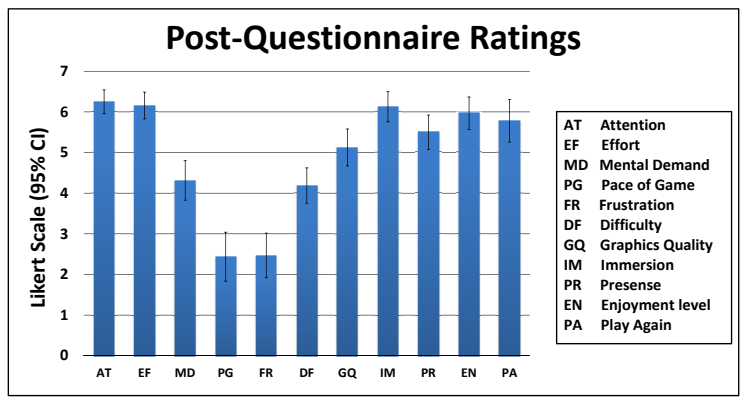

Figure 9. Mean ratings for the game post-questionnaire.

icance found between head tracking and stereoscopic 3D for necessity ratings. People did not think that stereoscopic 3D or head tracking is a necessity for future games. Except for minor eye strain, none of the participants noticed any symptoms (headache, dizziness, or nausea) from viewing games in stereoscopic 3D.

Out of all participants, 17 people thought that the depth perception was better with the presence of stereoscopic 3D, 17 people thought that it was more enjoyable to play with stereoscopic 3D, only 9 people thought that stereoscopic 3D helped them to judge the relative position of the enemies in the game, and 15 people thought that stereoscopic 3D made the game look more realistic. For the head tracking questionnaire, 17 people thought that head tracking added more realism to the game, 26 people thought that it was helping them to find enemies in the game environment, 26 people thought that it was much easier to look around with head tracking, and 25 people thought that zoom feature was helping them shoot distant enemies.

\section{Qualitative Results}

When asked about their experience playing the game, participants gave a variety of responses. One participant mentioned that he was very impressed with $3 \mathrm{D}$ effects in the game and it distracted him while playing. He occasionally felt like just enjoying the view rather than playing the game. Two of our participants were very sensitive to stereoscopic 3D and mentioned that it was very uncomfortable for them to play the game in 3D. One participant mentioned that it was uncomfortable to wear 3D glasses throughout the experiment. Two participants did not like zooming using head tracking because they felt uncomfortable being close to display screen while playing. Few participants mentioned that it was much easier 


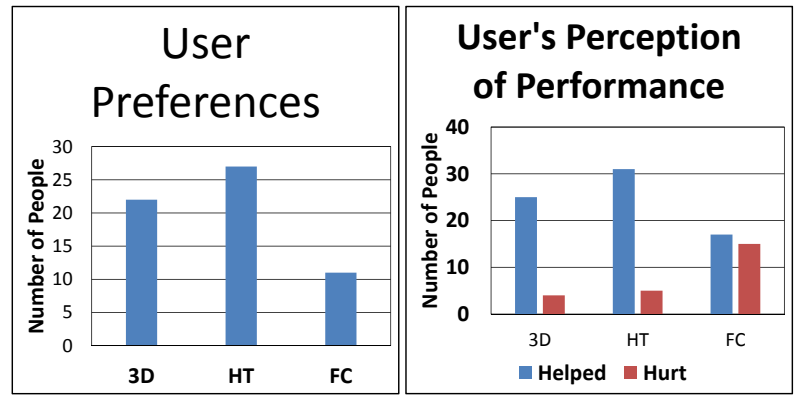

Figure 10. User preferences and user's perception of stereoscopic 3d, head tracking and finger-count shortcuts.

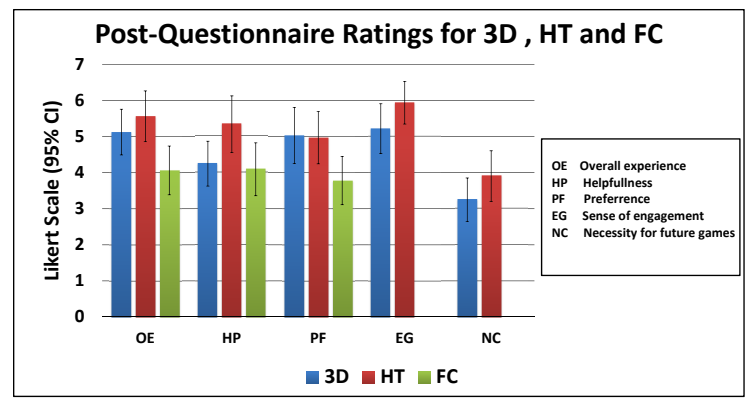

Figure 11. Mean ratings for the stereoscopic 3D, head tracking and finger-count post-questionnaire. Please note that EG and NC ratings were not collected for finger-count shortcuts.

to use finger-count shortcuts than buttons because they don't have to look down to find the button corresponding to a enemy color.

We got some interesting ideas when they were asked for other possible game tasks where finger-count gestures could be useful. One participant suggested to use finger-count gestures to quickly select and send pre-assigned text messages to other gamers in a multiplayer gaming environment. Currently, this task requires using a mouse which may not be the fastest choice. Another participant mentioned that finger-count gestures could be useful to solve some mini-puzzles, requiring selection from a set of items (e.g. Tower of Hanoi puzzle in Mass Effect where the task is to move blocks between towers), in games. A few other comments include using fingercount gestures to switch between different characters in a multi-character game (e.g. Trine), to switch between items in the minecraft game, to teleport a game character to different numbered locations in the game, and to assign a task (from a set of numbered tasks) to a game character.

\section{DISCUSSION}

We found significant performance benefits, in terms of number of enemies killed, due to combined usage of stereoscopic 3D, head tracking and finger-count shortcuts. Survival time was similar compared to the condition with monoscopic display, no head tracking, and button based weapon switch. Essentially, it means that people killed more enemies in the same time as in the condition with none of these technologies present. Therefore, the combined usage of the three technologies improved the performance of the users and we were able to accept out first hypothesis H1.
We did not find any significant performance differences based on display mode. These results are not surprising because prior experiments which studied effects of stereoscopic 3D [13, 19, 18] also found similar results. Kulshreshth et al. [11] found performance benefits of stereoscopic 3D for some video games (a pool table game and a game involving manipulation of 3D blocks) depending upon the user's prior gaming experience. But, those games were very different from our air-combat games and had tasks requiring precise motion in three dimensional space. For our game, the aircraft was moving in 3D space and enemies could be locked which does not require that much precision to shoot. Hence, we were not able to accept our second hypothesis $\mathrm{H} 2$.

Our experiment indicates that participants performed significantly better, in terms of enemies killed and survival time, when head tracking was present. Availability of head tracking helped participants find enemies faster in the environment without rotating the whole aircraft. When they were using button based head controls, it was not as easy to control the head as in case of head tracking which used natural head movements for controlling the player's head. Occasionally, while turning, participants used head tracking to make sure the turn is safe and would not end up in a collision with mountains or the enemies in the vicinity. Based on these results, we accepted our third hypothesis H3.

Most participants were positive about the game and felt an enhanced sense of engagement while playing when stereoscopic 3D and/or head tracking was present. They mentioned that depth perception, due to presence of stereoscopic 3D, made the game very realistic and more enjoyable. They felt as if they were actually flying that plane. Furthermore, they mentioned that it was very natural to use head tracking for searching enemies and it made the game very realistic. It was also mentioned that the gaming experience was best when both head tracking and stereoscopic 3D was present.

User's performance with finger-count shortcuts was as fast as with buttons and we were able to accept our fourth hypothesis H4. We expected these results based on the fact that the recognition time for our finger-count gestures (under a second) was approximately same as that of a button press. Moreover, it has already been shown that finger-count gestures are easy to learn and fast to perform [9]. Consequently, all participants were able to learn these gestures quickly and use them for weapon switching task in the game.

Interestingly, people were divided about their views on fingercount shortcuts. About half of participants preferred using finger-count shortcuts while another half did not. One possible reason could be familiarity with the button based interfaces (game controllers, keyboard/mouse, etc.) for video games. Most people play games using button based game controllers. Some of them like motion controlled games and some don't. Another possibility could be higher cognitive demand associated with finger-count shortcuts. In case of finger-count shortcuts, they need to control both hands independently and in different spatial areas requiring more cognitive attention than pressing buttons on a joystick. Consequently, we were unable to accept our fifth hypothesis H5. 
Gaming experience of participants did not play a significant role in performance across different gaming conditions. In prior experiments with stereoscopic 3D [11] and head tracking [10], it was found that gaming performance across different gaming conditions could be affected by gaming experience of the participants. Casual users focus more on playing the game rather than using added features (head tracking or stereoscopic 3D) to their advantage. Meanwhile, expert users try to use these additional features to improve their game play performance. Our air-combat game was an easy game to play and all participant were allowed to practice the game, for about 20-25 minutes, before the experiment began. This gave them ample time to get themselves familiar with the game and not worry about learning the game during the actual experiment. This could have been the reason we did not noticed any significant interactions based on the participants prior gaming experience.

Compared to prior research $[3,15]$, which studied interaction between head tracking and stereoscopic 3D, our results (see Figure 7) were slightly different. In our case, we found that head tracking significantly improved performance but stereoscopic 3D did not. Barfield et al. [3] found that display mode did not affect performance when head tracking was present and performance was better with stereoscopic 3D when head tracking was absent. Regan et al. [15] found that performance was best when stereoscopic 3D and head tracking was used together, but provided little benefit when used individually. One possible explanation of this difference could be the difference in the tasks presented to the participants as part of the experiment. Barfield et al. [3] used a wire tracing task, in which the objective was to move a virtual stylus (controlled by a real stylus) along the path of a virtual wire as quickly as possible without touching the wire. In case of [15], participants inspected cave structures with layers connected by vertical tubes and the task was to count the number of tubes connecting the horizontal layers. In both experiments, these were simple isolated tasks which were very different from playing a video game requiring a user to pay attention to many things while game play.

We found that people used head controls more often when head tracking was present. In case of button based head control, a combination of joystick hat switch and buttons (see Figure 3) was used to control the head of the player. On the other hand, when head tracking was present, natural head head movements were used to perform the same task. Cognitively, using buttons was much more difficult than using head tracking. Hence, while game play people refrained from using head controls frequently when head tracking was absent.

There are a few factors that could have affected our results. Our implementation of stereoscopic 3D, using dynamic stereo parameters, was different from all the past implementations. This could have a minor effect on our results but we still believe that the results will be similar or worse (due to less depth perception) with fixed stereo parameters. The size of display screen used could also have some influence on how much users can turn while using head tracking. The ideal choice would be very wide and curved display, with 180 degree field of view, but such stereoscopic 3D displays are not easily available. Most participants in our user study were males and this gender imbalance could have a minor effect on our results.

\section{CONCLUSION AND FUTURE WORK}

We presented an in-depth study which investigates how the combined use of several 3D user interface technologies affects the gaming experience. We designed an air-combat game keeping stereoscopic 3D, head tracking, and fingercount shortcuts usage in mind based on existing design ideas from the literature. A within subjects experiment was conducted where we examined game performance data, head tracking usage data, and data on user perception of the game. Our results show that people perform significantly better with the combined use of these technologies. Additionally, we found that head tracking was a major contributor to these performance benefits. The finger-count shortcuts also did not add much to the performance. However, about half of our participant preferred to use finger-count shortcuts compared to buttons for switching between weapons.

In our experiment we used head tracking data to dynamically optimize stereo parameters. But, head tracking provides only an approximate look direction and requires an user to rotate his/her head. In the future, we would like to use eye tracking to find where the user is looking and then optimize stereo parameters based on that information. Playing the game for different durations (e.g., 20 minutes, 40 minutes, or $60 \mathrm{~min}$ utes every other day for two weeks) might affect the game experience and would be interesting to look at in the future. We studied only a single game genre as part of our experiment. In the future, it would be interesting to explore these technologies for other game genres as well.

\section{ACKNOWLEDGMENTS}

This work is supported in part by NSF CAREER award IIS0845921 and NSF awards IIS-0856045 and CCF-1012056. We would also like to thank the other members of ISUE lab for their support and the anonymous reviewers for their useful comments and feedback.

\section{REFERENCES}

1. Bailly, G., Lecolinet, E., and Guiard, Y. Finger-count \& radial-stroke shortcuts: 2 techniques for augmenting linear menus on multi-touch surfaces. In Proceedings of the SIGCHI Conference on Human Factors in Computing Systems, CHI'10, ACM (New York, NY, USA, 2010), 591-594.

2. Bailly, G., Walter, R., Müller, J., Ning, T., and Lecolinet, E. Comparing free hand menu techniques for distant displays using linear, marking and finger-count menus. In Human-Computer Interaction INTERACT 2011, vol. 6947 of $L N C S$. Springer Berlin Heidelberg, 2011, 248-262.

3. Barfield, W., Hendrix, C., and Bystrom, K.-E. Effects of stereopsis and head tracking on performance using desktop virtual environment displays. Presence: Teleoper. Virtual Environ. 8, 2 (Apr 1999), 237-240.

4. Bowman, D. A., Kruijff, E., LaViola Jr, J. J., and Poupyrev, I. 3D User Interfaces: Theory and Practice. Addison-Wesley, 2004. 
5. Fisher, R. A. The design of experiments. Oxford, England: Oliver \& Boyd, 1935.

6. Fujimoto, M., and Ishibashi, Y. The effect of stereoscopic viewing of a virtual space on a networked game using haptic media. In Proceedings of the ACM SIGCHI international Conference on Advances in Computer Entertainment, ACM (New York, NY, 2005), 317-320.

7. Holm, S. A simple sequentially rejective multiple test procedure. Scandinavian Journal of Statistics 6, 2 (1979), 65-70.

8. Howarth, P. A. Potential hazards of viewing 3D stereoscopic television, cinema and computer games: a review. Ophthalmic \& Physiological Optics : The Journal of the British College of Ophthalmic Opticians (Optometrists) 31, 2 (2011), 111-122.

9. Kulshreshth, A., and LaViola, Jr., J. J. Exploring the usefulness of finger-based $3 \mathrm{~d}$ gesture menu selection. In Proceedings of the SIGCHI Conference on Human Factors in Computing Systems, CHI '14, ACM (New York, NY, USA, 2014), 1093-1102.

10. Kulshreshth, A., and LaViola Jr., J. J. Evaluating performance benefits of head tracking in modern video games. In Proceedings of the 1st symposium on Spatial user interaction, SUI '13, ACM (New York, NY, USA, 2013), 55-60.

11. Kulshreshth, A., Schild, J., and LaViola Jr., J. J. Evaluating user performance in 3D stereo and motion enabled video games. In Proceedings of the International Conference on the Foundations of Digital Games, ACM (New York, NY, 2012), 33-40.

12. LaViola, J. J., Forsberg, A. S., Huffman, J., and Bragdon, A. The influence of head tracking and stereo on user performance with non-isomorphic $3 \mathrm{~d}$ rotation. In Proceedings of the 14th Eurographics Conference on Virtual Environments, EGVE'08, Eurographics Association (Aire-la-Ville, Switzerland, Switzerland, 2008), 111-118.

13. Litwiller, T., and LaViola Jr., J. J. Evaluating the benefits of 3D stereo in modern video games. In Proceedings of the SIGCHI Conference on Human Factors in Computing Systems, CHI'11, ACM (New York, NY, USA, 2011), 2345-2354.

14. McMahan, R. P., Bowman, D. A., Zielinski, D. J., and Brady, R. B. Evaluating display fidelity and interaction fidelity in a virtual reality game. IEEE Transactions on Visualization and Computer Graphics 18, 4 (Apr. 2012), 626-633.

15. Ragan, E. D., Kopper, R., Schuchardt, P., and Bowman, D. A. Studying the effects of stereo, head tracking, and field of regard on a small-scale spatial judgment task. IEEE Transactions on Visualization and Computer Graphics 19, 5 (2013), 886-896.

16. Rajae-Joordens, R. J. Measuring experiences in gaming and tv applications. In Probing Experience, vol. 8 of Philips Re. Springer Netherlands, 2008, 77-90.
17. Schild, J., Bölicke, L., LaViola Jr., J. J., and Masuch, M. Creating and analyzing stereoscopic 3D graphical user interfaces in digital games. In Proceedings of the SIGCHI Conference on Human Factors in Computing Systems, CHI '13, ACM (New York, NY, USA, 2013), 169-178.

18. Schild, J., LaViola, Jr., J. J., and Masuch, M. Altering gameplay behavior using stereoscopic $3 \mathrm{~d}$ vision-based video game design. In Proceedings of the SIGCHI Conference on Human Factors in Computing Systems, CHI '14, ACM (New York, NY, USA, 2014), 207-216.

19. Schild, J., LaViola Jr., J. J., and Masuch, M. Understanding user experience in stereoscopic 3D games. In Proceedings of the SIGCHI Conference on Human Factors in Computing Systems, CHI '12, ACM (New York, NY, USA, 2012), 89-98.

20. Schild, J., and Masuch, M. Fundamentals of stereoscopic 3D game design. In ICEC, International Federation For Information Processing (2011), 155-160.

21. Sko, T., and Gardner, H. Head tracking in first-person games: Interaction using a web-camera. In Human-Computer Interaction - INTERACT 2009, vol. 5726 of Lecture Notes in Computer Science. Springer Berlin / Heidelberg, 2009, 342-355.

22. Sko, T., Gardner, H., and Martin, M. Studying a head tracking technique for first-person-shooter games in a home setting. In Human-Computer Interaction INTERACT 2013, vol. 8120 of Lecture Notes in Computer Science. Springer Berlin Heidelberg, 2013, 246-263.

23. Terlecki, M., and Newcombe, N. How important is the digital divide? the relation of computer and videogame usage gender differences in mental rotation ability. Sex Roles 53 (2005), 433-441.

24. Wang, S., Xiong, X., Xu, Y., Wang, C., Zhang, W., Dai, $X$., and Zhang, D. Face-tracking as an augmented input in video games: enhancing presence, role-playing and control. In Proceedings of the SIGCHI Conference on Human Factors in Computing Systems, CHI '06, ACM (New York, NY, USA, 2006), 1097-1106.

25. Ware, C. Dynamic stereo displays. In Proceedings of the SIGCHI Conference on Human Factors in Computing Systems, CHI '95, ACM Press/Addison-Wesley Publishing Co. (New York, NY, USA, 1995), 310-316.

26. Yim, J., Qiu, E., and Graham, T. C. N. Experience in the design and development of a game based on head-tracking input. In Proceedings of the 2008 Conference on Future Play: Research, Play, Share, Future Play '08, ACM (New York, NY, USA, 2008), 236-239.

27. You, J., Jiang, G., Xing, L., and Perkis, A. Quality of visual experience for 3D presentation - stereoscopic image. In High-Quality Visual Experience, M. Mrak, M. Grgic, and M. Kunt, Eds., Signals and Communication Technology. Springer Berlin Heidelberg, 2010, 51-77. 